\title{
Deriving Class Association Rules Based on Levelwise Subspace Clustering
}

\author{
Takashi Washio, Koutarou Nakanishi, and Hiroshi Motoda \\ I.S.I.R., Osaka University, 8-1, \\ Mihogaoka, Ibaraki City, \\ Osaka, 567-0047 Japan
}

\begin{abstract}
Most approaches of Class Association Rule (CAR) based classification have not intensively addressed the classification of instances including numeric attributes. In this paper, a levelwise subspace clustering method deriving hyper-rectangular clusters is proposed to efficiently provide quantitative, interpretative and accurate CARs.
\end{abstract}

\section{Introduction}

"Class Association Rules (CARs)" for classification has been proposed in recent studies [12]3]. These rules have the form " $\left\{<p_{1}: q_{1}>, \ldots,<p_{m}: q_{m}>\right\} \Rightarrow c l$ " where $\langle p: q\rangle$ is an item and $c l$ a class. $p$ represents an attribute and $q$ its value. An example is " $\{<$ Age : $[30,39]>,<$ Married : Yes $>,<$ NumCars : $[2,2]>\} \Rightarrow$ Houseowner" stating "A person who is in his/her thirties, married, and owns two cars belongs to the class Houseowner." Here, a "numeric item" has a numeric interval value whereas a "categorical item" has a categorical value. A numeric item $\left\langle p: q>\right.$ in the CAR is supported by a numeric item $\left\langle p_{t}: q_{t}\right\rangle$ in an instance $t$ if $p_{t}=p$ and $q_{t} \subseteq q$ where $\subseteq$ states that the range of $q_{t}$ is within the range of $q$. Hence, " $t_{1}=\{<$ Age : [35,37] $>,<$ Married: Yes $\rangle,\langle$ NumCars : $[2,2]>,<$ Child : $[3,3]>\}$ " supports the aforementioned rule body, whereas " $t_{2}=\{<$ Age $:[29,31]>,<$ Married : Yes $>,<$ NumCars $:[2,2]>,<$ Child: $[3,3]>\}$ " does not, because $<$ Age $:[29,31]>$ is not within $<$ Age $:[30,39]>$. Given a training data set $D$ which is a table (or a set) of class labeled instances (transactions), let $D_{c l}$ be a set of all instances having a class $c l$ in $D$. The body of a CAR including numeric items is a "quantitative frequent itemset," QFI in short, which is supported by $D_{c l}$ more frequently than a "minimum support (minsup)" threshold. The numeric part of a QFI corresponds to an axis-parallel and hyper-rectangular region in an attribute subspace of $D$.

CBA, CMAR and CAEP are the representatives of the CAR based classification 1213 . Especially CAEP, using the strength of all CARs, widely shows the best performance among many rule-based classifiers including C4.5. However, it discretizes each numeric attribute by an entropy measure without considering the dependency of the distributions among multiple attributes, and thus a cluster of instances having the same class can often be fragmented. An effective solution for this issue is the introduction of the clustering in every numeric attribute 
subspace to derive strong rule bodies. CLIQUE, DOC and SUBCLU seek dense clusters in every subspace [456. However, they do not provide QFIs covering both numeric and categorical attributes and corresponding to axis-parallel and hyper-rectangular clusters in an efficient manner. The approaches to mine quantitative association rules have addressed this issue [7/8. However, they discretize each attribute without considering the dependency among attributes, and thus can result in the aforementioned fragmentation.

In this study, a novel and efficient approach to the exhaustive, axis-parallel and hyper-rectangular subspace clustering is proposed. Moreover, the combined use of this clustering and CAEP is evaluated. The proposed clustering algorithm has a levelwise structure. While this is similar to SUBCLU, our approach can derive clusters on both numeric and categorical items, and the numeric items having interval values can be processed.

\section{CAEP}

CAEP is briefly explained at first 3]. The training phase of CAEP consists of two processes. The first is to derive all rule bodies. Let the support of an itemset $a$ by $D_{c l}$ be support $_{D_{c l}}(a)=\left|\left\{t \in D_{c l} \mid a \in t\right\}\right| /\left|D_{c l}\right|$. For every $c l$, a set of QFIs,

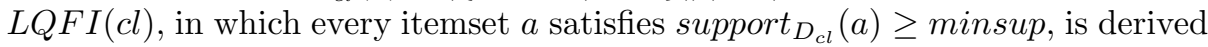
from $D_{c l}$. Subsequently, for every $a \in L Q F I(c l)$, the following "growth rate" for a class $c l$ is calculated. Let $\bar{D}_{c l}=D-D_{c l}$ be the opponent instances of $c l$.

\section{Growth rate:}

If support $\bar{D}_{c l}(a) \neq 0$, growth_rate $_{\bar{D}_{c l} \rightarrow D_{c l}}(a)=\frac{\text { support }_{D_{c l}}(a)}{\text { support }_{\bar{D}_{c l}}(a)}$, If support $_{\bar{D}_{c l}}(a)=0$ and support $_{D_{c l}}(a) \neq 0$, growth_rate $\bar{D}_{c l} \rightarrow D_{c l}(a)=\infty$, Otherwise growth_rate $\bar{D}_{c l} \rightarrow D_{c l}(a)=0$.

When the growth rate of $a$ is more than a "growth rate threshold" $\rho(>1)$, i.e., growth_rate $\bar{D}_{c l} \rightarrow D_{c l}(a) \geq \rho, a$ is called an "emerging pattern (EP)" and selected as a rule body where its head has the class $c l$, i.e., $a \Rightarrow c l$. Let $L E P(c l)$ be a set of all EPs selected from $L Q F I(\mathrm{cl})$ under this measure. The underlying principle here is to select the rule bodies having the strength to differentiate the class $c l$ from the others. Even if the rule confidence is high in $D_{c l}$, the rule can match many instances in $\bar{D}_{c l}$. Such rules are weak for classification.

The second process is to derive a "base score." First, the strength of an EP a based on the relative difference between support $_{D_{c l}}(a)$ and $\operatorname{support}_{\bar{D}_{c l}}(a)$ is introduced as support $_{D_{c l}}(a) /\left(\operatorname{support}_{D_{c l}}(a)+\operatorname{support}_{\bar{D}_{c l}}(a)\right)=$ growth_rate $_{\bar{D}_{c l} \rightarrow D_{c l}}(a) \quad /\left(\right.$ growth_rate $\left.\bar{D}_{c l} \rightarrow D_{c l}(a)+1\right)$. The following "aggregate score" represents the possibility of $t$ to be classified into $c l$ by EPs in $\operatorname{LEP}(\mathrm{cl})$.

\section{Aggregate score:}

$$
\text { score }(t, c l)=\sum_{a \subseteq t, a \in L E P(c l)} \frac{\text { growth_rate }(a)}{\text { growth_rate }(a)+1} * \text { support }_{D_{c l}}(a) .
$$

Because the number of EPs for each $c l$ may not be balanced, instances may get higher scores for some classes. A base score is introduced to eliminate this bias. 


\section{Base score:}

base_score $(\mathrm{cl})$ is the median of all aggregate scores in $\left\{\operatorname{score}(t, c l) \mid t \in D_{c l}\right\}$. The testing phase uses base_score $(c l)$, growth_rate(a) and support $_{D_{c l}}(a)$ obtained in the training phase. Given a test instance $t$, its aggregate score for $c l$, score $(t, c l)$, is computed from these results and Eq.(1). Then, it is normalized by base_score $(\mathrm{cl})$ to eliminate the aforementioned bias as follows.

\section{Normalized score:}

norm_score $(t, c l)=\frac{\text { score }(t, c l)}{\text { base_score }(c l)}$.

$c l$ having the maximum normalized score is assigned to the class of $t$. Except the derivation of $L Q F I(\mathrm{cl})$ for all $\mathrm{cl}$, the computational complexity of CAEP is $O(N)$ where $N=|D|$, since it scans the training data only twice.

\section{Mining Rule Bodies of CARs}

\subsection{Levelwise Subspace Clustering}

First we focus on the clustering of instances consisting of numeric items only. In our approach, the density of instances in a subspace is defined on their projected distribution to every attribute axis while ensuring the exhaustive finding of the clusters. The computational complexity is around $O(N \log N)$ as discussed later.

Let $t$ and $t^{\prime}$ be instances sharing a numeric attribute $p$ with interval values $q$ and $q$ ' respectively. The " $\Delta_{p}$-neighborhood" $N_{\Delta_{p}}(t)$ on $p$ is defined by $N_{\Delta_{p}}(t)=\left\{t^{\prime} \in D_{c l} \mid\right.$ Dist $\left._{p}\left(q, q^{\prime}\right) \leq \Delta_{p}\right\}$ where "permissible range" $\Delta_{p}$ is a real positive number on $p$. If intervals $q$ and $q^{\prime}$ overlap, then $\operatorname{Dist}_{p}\left(q, q^{\prime}\right)=0$, otherwise $\operatorname{Dist}_{p}\left(q, q^{\prime}\right)$ is the distance between their boundaries facing each other. An instance $t \in D_{c l}$ is called a "core instance" on $p$ if $N_{\Delta_{p}}(t)$ contains at least MinPts instances, i.e., $\left|N_{\Delta_{p}}(t)\right| \geq$ MinPts. When a core instance $t$ is contained in $N_{\Delta_{p}}\left(t^{\prime}\right)$ of another core instance $t^{\prime}, t$ and $t^{\prime}$ have a "connection."

Definition 1 (Density-Connected Set). A non-empty subset $C \subseteq D_{\text {cl }}$ is a "density-connected set" on $p$ if $C$ is the union of the $\Delta_{p}$-neighborhoods of core instances where all the core instances are in a chain of connections on $p$.

Definition 2 (Dense Cluster). A "dense cluster" $C^{S} \subseteq D_{c l}$ in a subspace formed by a set of numeric attributes $S$ is defined as a maximal set of instances which is a density-connected set on every $p \in S$ in $D_{c l}$.

Definition 3 (Quantitative Frequent Itemset). Let $C^{S} \subseteq D_{c l}$ be a dense cluster in a subspace $S$ and $a\left(C^{S}\right)=\left\{\langle p: q\rangle \mid p \in S, q=\left[\min _{p}\left(C^{S}\right)\right.\right.$, $\left.\left.\max _{p}\left(C^{S}\right)\right]\right\}$ an itemset where $\min _{p}\left(C^{S}\right)$ and $\max _{p}\left(C^{S}\right)$ are the minimum and the maximum interval boundaries of instances in $C^{S}$ on $p$. If $\left|C^{S}\right| \geq$ minsup, i.e., support ${ }_{D_{c l}}\left(a\left(C^{S}\right)\right) \geq$ minsup, then $a\left(C^{S}\right)$ is a "quantitative frequent itemset (QFI)." When the dimension of $S$ is $k$, it is called a $k$-QFI.

A QFI is a dense, axis-parallel and monotone hyper-rectangular region having a maximal volume in the subspace. Similarly to the dense clusters of SUBCLU, the following (anti-)monotonicity property of QFIs holds. 
Table 1. An example of transaction data set of $c l=$ Houseowner; $D_{\text {Houseowner }}$

\begin{tabular}{|c|c|}
\hline $\begin{array}{l}t_{1}=(\{<\text { Age: }[20,23] \\
t_{2}=(\{<\text { Age: }[30,30] \\
t_{3}=(\{<\text { Age: }[30,30] \\
t_{4}=(\{<\text { Age: }[30,35] \\
t_{5}=(\{<\text { Age: }[35,37]\end{array}$ & 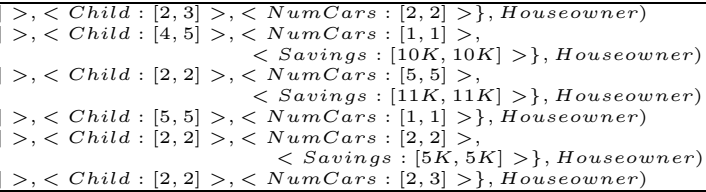 \\
\hline
\end{tabular}

Table 2. Process of levelwise subspace clustering of $D_{\text {Houseowner }}$

\begin{tabular}{|c|}
\hline $\begin{array}{l}\text { 1-QFIs } \\
\left(\{<\text { Age: }[30,39]>\},\left\{t_{2}, t_{3}, t_{4}, t_{5}, t_{6}\right\}\right),\left(\{<\text { Child: }[2,5]>\},\left\{t_{1}, t_{2}, t_{3}, t_{4}, t_{5}, t_{6}\right\}\right) \\
\left(\{<\text { NumCars : }[1,3]>\},\left\{t_{1}, t_{2}, t_{4}, t_{5}, t_{6}\right\}\right),\left(\{<\text { Savings }:[10 K, 11 K]>\},\left\{t_{2}, t_{3}\right\}\right)\end{array}$ \\
\hline $\begin{array}{l}\text { 2-QFIs } \\
\left(\{<\text { Age }:[30,39]>,<\text { Child }:[2,2]>\},\left\{t_{3}, t_{5}, t_{6}\right\}\right) \\
\left(\{<\text { Age }:[30,35]>,<\text { Child }:[4,5]>\},\left\{t_{2}, t_{4}\right\}\right) \\
\left(\{<\text { Age }:[30,39]>,<\text { NumCars: }[1,3]>\},\left\{t_{2}, t_{4}, t_{5}, t_{6}\right\}\right) \\
\left(\{<\text { Age : }[30,30]>,<\text { Savings: }[10 K, 11 K]>\},\left\{t_{2}, t_{3}\right\}\right) \\
\left(\{<\text { Child }:[2,5]>,<\text { NumCars }:[1,3]>\},\left\{t_{1}, t_{2}, t_{4}, t_{5}, t_{6}\right\}\right)\end{array}$ \\
\hline $\begin{array}{l}\text { 3-QFIs } \\
\left(\{<\text { Age }:[35,39]>,<\text { Child }:[2,2]>,<N \text { umCars }:[2,3]>\},\left\{t_{5}, t_{6}\right\}\right) \\
\left(\{<\text { Age }:[30,35]>,<\text { Child }:[4,5]>,<N \text { umCars }:[1,1]>\},\left\{t_{2}, t_{4}\right\}\right)\end{array}$ \\
\hline
\end{tabular}

Lemma 1 (Monotonicity). $\forall T \subseteq S$, if $a\left(C^{S}\right)$ is a $Q F I$ in $S$, then a $Q F I$ $a\left(C^{T}\right)$ supported by $a\left(C^{S}\right)$, i.e., $a\left(C^{S}\right) \subseteq a\left(C^{T}\right)$, exists in $T$.

Proof. Because $C^{S}$ is a density-connected set on $\forall p \in S$, it is a densityconnected set on $\forall p \in T$, and hence $C^{S} \subseteq C^{T}$. Therefore, $\forall p \in T,\left[\min _{p}\left(C^{S}\right)\right.$, $\left.\max _{p}\left(C^{S}\right)\right] \subseteq\left[\min _{p}\left(C^{T}\right), \max _{p}\left(C^{T}\right)\right]$, and $a\left(C^{T}\right)$ is supported by $a\left(C^{S}\right)$.

Accordingly, a levelwise bottom up approach is applicable to search all QFIs. We exemplify its operation by using the dataset in Table 1. Each instance (transac-

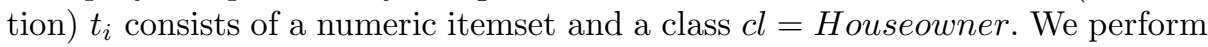
the clustering under parameters of $\Delta_{\text {Age }}=5, \Delta_{\text {Child }}=1, \Delta_{\text {NumCars }}=1$, $\Delta_{\text {Savings }}=1 \mathrm{~K}$, MinPts $=1$ and minsup $=2$. First, the items in each $t_{i}$ are lexicographically ordered by the attribute names. This has been already done in this table. Subsequently, 1-QFIs are searched, where the instances are maximally density-connected on an attribute. For Age, a 1-QFI, $\{<$ Age : [30, 39] $\rangle\}$, exists since the items densely range from 30 to 39 under $\Delta_{A g e}=5$, and its support 5 is more than minsup. This 1-QFI with its "transaction id list (TID-List)" is indicated in Table 2, Each attribute has an 1-QFI in this example.

In the next step, the levelwise search for $k$-QFIs $(k>1)$ starts. Index lists named TID - List are used to point instances in $D_{c l}$ similarly to AprioriTid algorithm [9]. Assuming that all $(k-1)$-QFIs are known, the following "CandidateGeneration" operation derives all candidate $k$-QFIs.

Definition 4 (Candidate-Generation).

Join Phase: For two $(k-1)$-QFIs sharing $k-2$ attributes,

$$
\begin{aligned}
& \left((k-1)-Q F I=\left\{<p_{1}: q_{1}>,<p_{2}: q_{2}>, \ldots,<p_{k-2}: q_{k-2}>,<p_{k-1}: q_{k-1}>\right\}, \text { TID }- \text { List }\right), \\
& \left((k-1)-Q F I^{\prime}=\left\{<p_{1}: q_{1}^{\prime}>,<p_{2}: q_{2}^{\prime}>, \ldots,<p_{k-2}: q_{k-2}^{\prime}>,<p_{k}: q_{k}^{\prime}>\right\}, \text { TID }- \text { List }^{\prime}\right),
\end{aligned}
$$

their join is derived as follows:

(candidate $-k-Q F I=\left\{<p_{1}: q_{1}^{c}>, \ldots,<p_{k-1}: q_{k-1}^{c}>,<p_{k}: q_{k}^{c}>\right\}, T I D-$ List $\left.^{c}\right)$. 
QFI-Count(candidate $\left.-k-Q F I, T I D-L i s t^{c}\right)$;

(1) $k-Q F I S=\phi, T I D L S=\phi$;

(2) If $\left|T I D-L i s t^{c}\right|<$ minsup return $k-Q F I S$;

(3) $S=\{p \mid\langle p: q\rangle$

$\in$ candidate $-k-Q F I, p$ is numeric. $\}$;

(4) $T I D L S . t e m p=\left\{T I D-\right.$ List $\left.^{c}\right\}$;

(5) while TIDLS $\neq$ TIDLS.temp do begin

(6) $T I D L S=T I D L$ S.temp;

(7) forall $p \in S$ do begin

(8) TIDLS.temp $=$

(9) end

MDCS $(T I D L$ S.temp, $p)$;

(10) end

(11) forall TID - List $\in$ TIDLS do begin

(12) $k-Q F I S=k-Q F I S+$

(13) end $(Q F I(S, T I D-L i s t), T I D-L i s t)$;

(14) return $k-Q F I S$;

Fig. 1. Algorithm of QFI-Count
(1) For each numeric attribute, create an index list sorted with the ascending order of $D$. Sort items in each $t \in D$ lexicographically.

(2) $L_{1}=\{(1-Q F I, T I D-$ List $)\}$;

(3) for $\left(k=2 ; L_{k-1} \neq \phi ; k++\right)$ do begin

(4) $\quad C_{k}=$ $\left\{\left(\right.\right.$ candidate $\left.\left.-k-Q F I, T I D-L i s t^{c}\right)\right\}=$ Extended - CandidateGeneration $\left(L_{k-1}\right)$;

(5) forall (candidate $-k-Q F I$,

(6) $\quad L_{k}=L_{k} \cup$ $L_{k}=L_{k} \cup$
$Q F I-C o u n t$ (candidate $-k-Q F I$

(7) end TID - List $\left.^{c}\right)$

(8) end

(9) Answer $L=\bigcup_{k} L_{k}$;

Fig. 2. Entire algorithm

where $q_{i}^{c}$ is the intersection of the two intervals $q_{i} \cap q_{i}^{\prime}$ for $i=1, \ldots, k-2$, $q_{k-1}^{c}=q_{k-1}, q_{k}^{c}=q_{k}^{\prime}$ and TID - List $^{c}=$ TID - List $\cap$ TID - List ${ }^{\prime}$. If some $q_{i}^{c}=\phi$, the two $(k-1)$-QFIs are not joined.

Prune Phase: For all $(k-1)$-subsets s of this candidate- $k$-QFI, if the following $(k-1)$-QFI exists:

$$
\forall<p_{i}: q_{i}^{c}>\in s, \exists<p_{i}: q_{i}>\in(k-1)-Q F I, q_{i}^{c} \cap q_{i} \neq \phi,
$$

the candidate-k-QFI is retained, and TID - List ${ }^{c}$ is a candidate dense cluster $\hat{C}^{S}$ where $|S|=k$. Otherwise the candidate- $k$-QFI is pruned.

This prune phase is based on Lemma 1, As far as $q_{i}^{c}$ intersects with $q_{i}$ in Eq.(2), the possibility that $s$ and $(k-1)-Q F I$ shares more than minsup transactions is not negligible. Thus the candidate $k$-QFI is retained under this condition. In Table 2, a candidate-2-QFI, $\{<$ Age : $[30,39]>,<$ Child : $[2,5]>\}$ with TID - List $^{c}=\left\{t_{2}, t_{3}, t_{4}, t_{5}, t_{6}\right\}$ is derived from two 1-QFIs, $\{<$ Age $\left.:[30,39]\rangle\right\}$ and $\{<$ Child: $[2,5]\rangle\}$. This passes the prune phase.

"QFI-Count" shown in Fig 1 derives dense clusters $C^{S}=T I D-$ List and their corresponding $k$-QFIs, if they exist, by assessing the density of instances in $\hat{C}^{S}$ based on Definition 2 and 3 . In the inside loop from (7) to (9), a maximal density-connected set $C$ is searched on $p$ within $\hat{C}^{S}$ at first in a function $M D C S$ along with Definition 1 under given $\Delta_{p}$ and MinPts. Multiple $C$ can be found when multiple dense clusters are included in $\hat{C}^{S}$. MDCS repeats to update $C$ on $p$ from every $C$ derived and kept in TIDLS.temp at the previous loop iteration. $C$ having a size less than minsup is discarded in MDCS. This update continues in the outer loop from (5) to (10), until each $C$ converges to $C^{S}$ where each $C^{S}$ is independent of the convergence process due to the (anti-)monotonicity property. In the loop from (11) to (13), each QFI corresponding to $C^{S}=$ TID - List is computed by Definition 3 in a function $Q F I$ and returned as the output. In the example, the candidate-2-QFI, $\{<$ Age : $[30,39]\rangle,\langle$ Child $:[2,5]\rangle\}$ 
with $T I D-$ List $^{c}=\left\{t_{2}, t_{3}, t_{4}, t_{5}, t_{6}\right\}$ is given to this QFI-Count. In the inside loop, MDCS derives TIDLS.temp $=\left\{\left\{t_{2}, t_{3}, t_{4}, t_{5}, t_{6}\right\}\right\}$ on Age. Next, it derives $T I D L S . t e m p=\left\{\left\{t_{3}, t_{5}, t_{6}\right\},\left\{t_{2}, t_{4}\right\}\right\}$ on Child. Further applications of MDCS do not change TIDLS.temp. Since the sizes of candidates are more or equal to minsup $=2$, two 2-QFIs, $\left(\{<\right.$ Age $:[30,39]>,<$ Child $\left.:[2,2]>\},\left\{t_{3}, t_{5}, t_{6}\right\}\right)$ and $\left(\{<\right.$ Age $:[30,35]>,<$ Child : $\left.[4,5]>\},\left\{t_{2}, t_{4}\right\}\right)$, are derived.

\subsection{Deriving QFIs of Numeric and Categorical Items}

Candidate-Generation is extended to derive QFIs consisting of numeric and categorical items. The categorical items in the joined itemset are given in the same way as in the AprioriTid algorithm. In the join phase of Definition 4, if $q_{i}^{c}=\phi$ for some numeric item or $q_{i} \neq q_{i}^{\prime}$ for some categorical item, the two given $(k-1)$ QFIs are not joined. Otherwise they are joined as $q_{i}^{c}=q_{i} \cap q_{i}^{\prime}$ for each numeric item and $q_{i}^{c}=q_{i}=q_{i}^{\prime}$ for each categorical item. In the prune phase, the condition $q_{i}^{c}=q_{i}$ for a categorical item is applied in addition to $q_{i}^{c} \cap q_{i} \neq \phi$ for a numeric item in Eq.(2). The algorithm QFI-Count of Fig 1 is also altered. When the candidate- $k$-QFI consists of categorical items only, the loop from (5) to (10) is skipped, and TIDLS $=$ TIDLS.temp is applied. The function $Q F I$ at step (12) is also altered. For a categorical attribute $p_{i}$, its value is set to be $q_{i}^{c}=q_{i}=q_{i}^{\prime}$.

The entire algorithm to derive QFIs from $D$ is indicated in Fig 2, Required parameters are $\Delta_{p}$ for all numeric attributes, MinPts and minsup. First, some index lists are created for the efficient processing in Extended-CandidateGeneration and QFI-Count. Subsequently, all QFIs are computed in $L$ by the adaptation of the AprioriTid Algorithm. In the implementation, the inversed indexing $\left(t_{i},\{\right.$ candidate $\left.-k-Q F I\}\right)$ from each $t_{i}$ to its containing candidate$k$-QFIs is used instead of (candidate $-k-Q F I, T I D-$ List $\left.^{c}\right)$ similarly to the standard AprioriTid. This approach is applied to $D_{c l}$ of every class $c l$ to derive $L Q F I(c l)$ required by CAEP described in the previous section.

\section{Experimental Evaluation}

\subsection{Computational Efficiency}

The most expensive task is the derivation of QFIs for CAR's bodies. Thus, its computational efficiency is evaluated by using artificial data sets. First, a set of seed items, $S S I$, is randomly generated where $r_{n} \%$ of them are numeric and the rest categorical. Second, a set of seed QFIs, $S Q F I$, is generated by randomly selecting seed items from $S S I$. The size of each QFI is determined by uniform random distribution having its average at $\overline{|Q F I|}$. Third, a set of instances (transactions) $D$ is generated where each instance $t$ is made by randomly selecting a $Q F I$ from $S Q F I$ and further randomly adding extra $2 \overline{|Q F I|}$ seed items taken from $S S I$ in the average. Finally, the values of numeric items in each $t$ are distorted by introducing Gaussian noise having 5\% amplitude. Our algorithm is tested on a Pentium 4 2.7 GHz PC with 2GB RAM. The default parameters for the test are $|S S I|=1000, r_{n}=50 \%,|S Q F I|=10, \overline{|t|}=12, N=|D|=40000$, 
Table 3. Complexity of clustering

\begin{tabular}{|l|l|c|c|}
\hline Para- & Range of & \multicolumn{2}{|c|}{ Dependency of } \\
meter & Assessment & Comp. Time & Mem.Cons. \\
\hline$|S S I|$ & {$[20,20000]$} & constant & constant \\
$r_{n}$ & {$[0 \%, 100 \%]$} & constant & constant \\
$|S Q F I|$ & {$[1,50]$} & $O(|S Q F I|)$ & $O(|S Q F I|)$ \\
$|\bar{t}|$ & {$[8,100]$} & exp. inc. & exp. inc. \\
minsup & {$[0.2 \%, 10 \%]$} & exp. dec. & exp. dec. \\
$\Delta_{p}$ & {$[0.1 \%, 100 \%]$} & inc. const. & inc. const. \\
MinPts & {$[1,8000]$} & dec. const. & dec. const. \\
$N$ & {$\left[200,10^{6}\right]$} & $O(N$ log $N)$ & $O(N)$ \\
\hline
\end{tabular}

exp. inc./dec. : exponential increase/decrease. inc./dec. const. : increase/decrease and saturation.

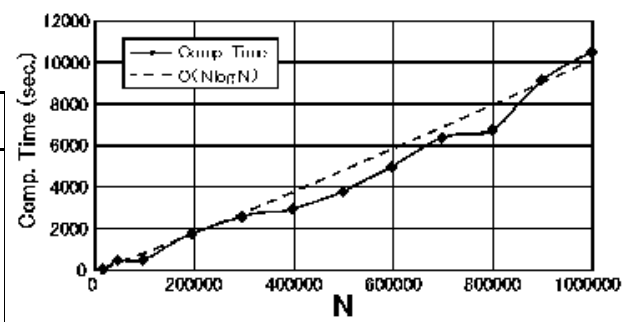

Fig. 3. $N$ vs. Comp. Time

minsup $=5 \%$, MinPts $=1$ and $\Delta_{p}=20 \%$ (in relative width of the maximum and minimum values of the instances on each numeric attribute).

The qualitative dependencies of computation time and memory consumption on the parameters summarized in Table 3 are similar to the AprioriTid. Their rapid increases are observed under the small values of $\Delta_{p}$ and MinPts where the values are comparable with the average gap among two different instances values. The dependency of the computation time on $N$ shown in Fig 3 is almost $O(N \log N)$ up to 1 million instances, while SUBCLU requiring the range query of each instance shows $O\left(N^{2}\right)$ where a dense cluster is such that for each instance in the dense cluster the neighborhood of a given radius $\epsilon$ has to contain at least a minimum number of MinPts instances [6]. The memory consumption is $O(N)$, because the size of the inversed TID-List is proportional to $N$.

\subsection{Classification Performance and Interpretability}

CAEP combined with QFI derivation is called LSC-CAEP in this paper. Its $10 \mathrm{CV}$ accuracy has been compared with C4.5, CBA, CMAR and the original CAEP by using the UCI repository data as indicated in Table 4. These data sets were selected so that the accuracy by various approaches has been reported [123]. The parameters of LSC-CAEP was set as minsup $=12 \%$, MinPts $=1, \Delta_{p}=16 \%$ and $\rho=1.1$, where their optimality has been confirmed through empirical surveys. The bold faces in the table are the best. The standard deviations of the accuracies over $10 \mathrm{CV}$ by C4.5, CBA and LSC-CAEP are $2.6 \%, 3.4 \%$ and $4.0 \%$ respectively, while the average discrepancies of the accuracies of LSC-CAEP are $5.6 \%$ from C4.5 and $4.5 \%$ from CBA in Table 4 . LSC-CAEP performs moderately better than C4.5 and better or equal at least to CBA. As shown in the last column of Table 4 the computation times of LSCCAEP ranges from 0.1 to $87 \mathrm{sec}$, while $\mathrm{C} 4.5$ and $\mathrm{CBA}$ are from 0.3 to $2.2 \mathrm{sec}$. The speed of LSC-CAEP usually remains practical due to its aforementioned good scalability, though it is affected by the dependency among attributes similarly to the Apriori algorithm.

The interpretability of the rules is important but a quite subjective matter. The following two QFIs having large support values are found by LSC-CAEP in Labor Relations Database in UCI repository. 
Table 4. Comparison of accuracies

\begin{tabular}{|c|c|c|c|c|c|c|c|c|}
\hline dataset & $\begin{array}{c}\text { num. of } \\
\text { records }\end{array}$ & $\begin{array}{c}\text { num. of } \\
\text { attributes(numeric) }\end{array}$ & $\begin{array}{c}\text { num. of } \\
\text { classes }\end{array}$ & C4.5 & CBA & CMAR & CAEP & $\begin{array}{c}\text { LSC-CAEP } \\
\text { [comp. time (sec) }]\end{array}$ \\
\hline Cleve & 303 & $13(5)$ & 2 & .782 & $\mathbf{. 8 2 8}$ & .822 & .833 & $.789[38]$ \\
\hline Ecoli & 336 & $8(7)$ & 8 & .824 & - & - & - & $\mathbf{. 8 3 1}[22]$ \\
\hline Heart & 270 & $13(6)$ & 2 & .808 & .819 & .822 & .837 & $\mathbf{. 8 4 5}[87]$ \\
\hline Hepatitis & 155 & $19(6)$ & 2 & .806 & .818 & .805 & .830 & $\mathbf{. 8 5 2}[26]$ \\
\hline Iris & 150 & $4(4)$ & 3 & .953 & .947 & .940 & .947 & $\mathbf{. 9 6 7}[0.1]$ \\
\hline Glass & 214 & $9(9)$ & 7 & .687 & $\mathbf{. 7 3 9}$ & .701 & - & $.681[19]$ \\
\hline Labor & 57 & $16(8)$ & 2 & .793 & .863 & .897 & - & $\mathbf{. 9 4 3}[0.1]$ \\
\hline Wine & 178 & $13(13)$ & 3 & .927 & .950 & .950 & .971 & $\mathbf{. 9 7 2}[52]$ \\
\hline Zoo & 101 & $16(0)$ & 7 & .922 & .968 & $\mathbf{. 9 7 1}$ & - & $.911[19]$ \\
\hline
\end{tabular}

support=19: $\{$ class:good, duration-years: $[2,2]$, working-hours: $[33,40]$, wage-inc.-2nd-year(\%):[4.0,5.8]\} . support $=16$ : $\{$ class:good, duration-years: $[3,3]$, working-hours: $[35,40]$, wage-inc.-2nd-year $(\%):[3.5,5.0]\}$. These QFIs suggest an assumption that the increase of job stability from 2 years to 3 years balances with admitting slightly longer working hours and $0.5 \%$ $0.8 \%$ less wage increase. The following two CARs having high growth rates are found in Iris data. The insights on the species of iris can be learned.

growth rate=4.5: petal width:[1.4-2.5]) $\rightarrow$ class:virginica

growth rate=1.9: sepal length:[4.9-7.0], sepal width:[2.0-3.4] $\rightarrow$ class:setosa.

The fine granularity of the interval boundaries helps the interpretation.

\section{Discussion and Conclusion}

To check the applicability of LSC-CAEP to large data sets, LSC-CAEP, C4.5 and CBA were applied to Census-Income data containing 199523 instances and 40 attributes (numeric: 7 categorical: 33) in UCI KDD Archive, and confirmed that the accuracy achieved by LSC-CAEP is $92.4 \%$, which is comparable with $94.3 \%$ and $94.0 \%$ of $\mathrm{C} 4.5$ and CBA respectively. Further improvement of the performance of LSC-CAEP will be addressed in future study.

The most expensive tasks in LSC-CAEP are the sort which is $O(N \log N)$ and QFI-Count of Fig 1. The maximal density-connected sets on every numeric attribute $p$ are easily derived in one scan of TIDLS.temp in MDCS by using the sort index list built at the first step in Fig 2. Hence it is $O(N)$ at maximum. The iteration of the outer loop from (5) to (10) in QFI-Count varies extensively. In the worst case, an instance is removed in each loop path from the edge of a region where instances are ranged in a periodic manner, and the loop becomes $O\left(N^{2}\right)$. However, in the most likely case which is an exponential density distribution, a portion $0<r<1$ of the instances in the average are retained in each loop path. The loop finishes by the time $r^{m} N$ becomes less than minsup where $m$ is the number of loop paths. Thus minsup $\leq r^{m} N$, and $m$ is around $O(\log N)$. Accordingly, the entire algorithm is expected to be $O(N \log N)$.

Our proposal enabled efficient subspace clustering on the mixtures of numeric and categorical data in a levelwise algorithm. Further new approaches of clustering and classification for large data sets can be developed along this line. 
Acknowledgement. The authors wish to thank Dr. Alexandre Termier in ISIR, Osaka Univ. for his extensive support to write this paper. This research was partially supported by the Japan Society for the Promotion of Science (JSPS) Grant-in-Aid for Scientific Research (B), 16300045, 2005.

\section{References}

1. Liu, B., Hsu, W., Ma, Y.: Integrating classification and association rule mining. Proc. of Fourth International Conference on Knowledge Discovery and Data Mining (1998)

2. Li, W., Han, J., Pei, J.: Cmar: Accurate and efficient classification based on multiple class-association rules. Proc. of First IEEE International Conference on Data Mining (2001) 369-376

3. Dong, G., Zhang, X., Wong, L., Li, J.: Caep: Classification by aggregating emerging patterns. Proc. of Second International Conference on Discovery Science, Lecture Notes in Computer Science 1721 (1999) 30-42

4. Agrawal, R., Gehrke, J., Gunopulos, D., Raghavan, P.: Automatic subspace clustering of high dimensional data for data mining applications. Proc. of the 1998 ACM SIGMOD international conference on Management of data (1998) 94-105

5. Procopiuc, C.M., Jones, M., Agarwal, P.K., Murali, T.M.: A monte carlo algorithm for fast projective clustering. Proceedings of the 2002 ACM SIGMOD international conference on Management of data (2002) 418-427

6. Kailing, K., Kriegel, H.P., Kroger, P.: Density-connected subspace clustering for high-dimensional data. Proc. Fourth SIAM International Conference on Data Mining (SDM'04) (2004) 246-257

7. Srikant, R., Agrawal, R.: Mining quantitative association rules in large relational tables. Proc. of 1996 ACM SIGMOD Int. Conf. on Management of Data (1996) $1-12$

8. Wang, K., Hock, S., Tay, W., Liu, B.: Interestingness-based interval merger for numeric association rules. Proc. of 4th Int. Conf. on Knowledge Discovery and Data Mining (KDD) (1998) 121-128

9. Agrawal, R., Srikant, R.: Fast algorithms for mining association rules. Proc. of 20th Int. Conf. on Very Large Data Bases (VLDB) (1994) 487-499 